\title{
Escala da Satisfação com a decisão em saúde: instrumento adaptado e validado para língua portuguesa
}

\author{
Decision making satisfaction in health scale: instrument adapted and validated to portuguese \\ Escala de la satisfacción con las decisiones en la salud: instrumento adaptado \\ y validado para el idioma portugués
}

\author{
Maria Júlia Costa Marques Martinho', Maria Manuela Ferreira Pereira da Silva Martins', Margareth Angelo" \\ 'Escola Superior de Enfermagem do Porto. Porto, Portugal. \\ "Universidade de São Paulo, Escola de Enfermagem, \\ Departamento de Enfermagem Materno-Infantil e Psiquiátrica. São Paulo-SP, Brasil.
}

Submissão: 30-07-2013 Aprovação: 16-09-2014

\section{RESUMO}

A tomada de decisão é uma área de investigação na saúde que tem vindo a ganhar importância quer pelos modelos de parceria de cuidados que dão protagonismo ao paciente e família, quer pela preocupação crescente com a qualidade e satisfação do cliente com os cuidados disponibilizados. Assim, propusemo-nos efetuar a adaptação transcultural e avaliar as propriedades psicométricas da versão portuguesa da "The Satisfaction with Decision Scale" de Holmes-Rovner (1996), que visa avaliar a satisfação com as decisões tomadas em saúde. A amostra foi constituída por 521 estudantes da Escola Superior de Enfermagem do Porto. Os resultados obtidos nos testes de fiabilidade revelam uma boa consistência interna para o total dos itens (Alpha Cronbach $=0,88$ ). O estudo psicométrico permite-nos afirmar que a versão em Português da "The Satisfaction with Decision Scale", que denominamos "Escala da Satisfação com a Decisão em Saúde", é um instrumento fidedigno e válido.

Descritores: Teoria da Decisão; Satisfação Pessoal; Pacientes; Cuidados de Enfermagem; Psicometria.

\begin{abstract}
Decision making is an area of health research that has gained importance both for the partnership models of care that give prominence to the patient and family, either by growing concern about quality and customer satisfaction with the care provided. So we decided to make the cultural adaptation and to evaluate the psychometric properties of the Portuguese version "The Satisfaction with Decision Scale" de Holmes-Rovner (1996), which aims to assess satisfaction with the decisions taken in health. The sample consisted of 521 nursing students the School of Nursing of Porto. The results of reliability tests show good internal consistency for the total items (Alpha Cronbach $=0.88$ ). The psychometric study allows us to state that the Portuguese version of "The Satisfaction with Decision Scale", we call "Escala da Satisfação com a Decisão em Saúde", is an instrument comparable with the original in terms of validity and reliability.
\end{abstract}

Key words: Decision Theory; Personal Satisfaction; Patients; Nursing Care; Psychometrics.

\section{RESUMEN}

La toma de decisiones es un área de investigación en salud que ha ganado importancia tanto por los modelos de atención dirigida al paciente y su familia, como por la creciente preocupación por la calidad y satisfacción del cliente con la atención recibida. Por esta razón decidimos hacer la adaptación transcultural y evaluar las propiedades psicométricas de la versión portuguesa "The Satisfaction with Decision Scale" de Holmes-Rovner (1996), que tiene como objetivo evaluar la satisfacción con las decisiones adoptadas en materia de salud. La muestra consta de 521 estudiantes de la Escuela de Enfermería del Porto. Los resultados de las pruebas de fiabilidad muestran una buena consistencia interna para la escala total (Alpha Cronbach = 0,88). El estudio psicométrico nos permite afirmar que la versión en portugués de "The Satisfaction with Decision Scale", que nosotros Ilamamos "Escala da Satisfação com a Decisão em Saúde", es válida.

Palabras clave: Teoría de las Decisiones; Satisfacción Personal; Pacientes; Atención de Enfermería; Psicometría. 


\section{INTRODUÇÃO}

A satisfação do paciente tem sido um elemento importante na avaliação dos cuidados de saúde ${ }^{(1-4)}$, particularmente para a Enfermagem, possibilitando-Ihe perceber o impacto das suas intervenções e a qualidade dos seus cuidados. A satisfação está associada com os melhores resultados em saúde, sendo um preditor importante do comportamento em saúde, como a adesão ao regime terapêutico instituído. Os pacientes, quando recorrem ao sistema de saúde, vão providos de experiências anteriores, que, associadas com a instrução e informação fornecidas pelos profissionais de saúde lhes permite definir a situação que estão a vivenciar, identificando assim as suas necessidades. O aumento progressivo da variedade de opções disponibilizadas aos doentes, no âmbito da saúde, têm tornado mais complexo o processo de tomada de decisão, cabendo aos profissionais de saúde o apoio necessário na escolha da alternativa mais satisfatória. Uma informação com alta qualidade, disponibilizada através do aconselhamento, ajuda os pacientes a compreender os riscos potenciais, os benefícios e incertezas das opções clinicas, auxiliando-os a escolher a opção que melhor se acomoda às suas preferências pessoais.

Os resultados dos estudos ${ }^{(5-7)}$ sugerem que os pacientes que são apoiados nas decisões de saúde são mais propensos a requererem informação, têm expectativas de resultado realistas, participam ativamente na tomada de decisão e têm níveis baixos de conflito nas decisões. A satisfação com os cuidados de enfermagem traduz, então, a convergência entre as expectativas do paciente de um cuidado ideal e a sua perceção da experiência individual do cuidado real prestado. Assim, a satisfação, pode ser descrita como um julgamento subjetivo que reflete o grau em que as escolhas efetuadas são consistentes com os valores de cada um $^{(8)}$. Algumas investigações $^{(9-12)}$ apontam determinados fatores como a educação, a idade, as experiências, as expectativas, o estado de saúde como influenciadores da satisfação dos pacientes.

Sendo também evidenciada, uma relação entre a satisfação do paciente e a competência e experiência clinica do enfermeiro ${ }^{(13-14)}$, dependendo o nível de satisfação do paciente, do grau em que os enfermeiros podem atender às suas necessidades e expetativas ${ }^{(13,15)}$.

Consideramos que dispor de um instrumento como medida de resultado para intervenções concebidas para apoiar os pacientes na tomada de decisão é de suma importância para a prática em saúde. A utilização de instrumentos com a finalidade de medir a satisfação do paciente na área da saúde teve início na década de 70 variando conforme os pressupostos que são feitas quanto ao que significa a satisfação(16).

A nossa opção por esta escala, "The Satisfaction with Decision Scale - (SWD)"(17) centrou-se no facto de esta ter sido construída com base num modelo conceptual que considera a decisão implementada eficaz, quando esta é informada e coerente com os valores do decisor, sendo a satisfação com a decisão medida independentemente do prognóstico ser bom ou $\mathrm{mau}^{(17)}$, contrariando muitas das escalas disponíveis, que não são sustentados por modelos teóricos ${ }^{(16)}$. Aliou-se também a este fato, a escala ser fácil de aplicar, já que é curta, podendo ser utilizada em vários contextos de cuidados de saúde. Para estabelecer a fiabilidade inicial do instrumento, a autora realizou um estudo piloto com uma amostra de conveniência de muIheres $(n=120)$ recrutadas do corpo docente e pessoal da $\mathrm{Mi}$ chigan State University, tendo o instrumento apresentado uma boa consistência interna, com um alfa de Cronbach $=0,88$. Posteriormente, a escala foi utlizada em vários estudos, designadamente, na avaliação das intervenções de apoio às mulheres na tomada de decisão, acerca da terapia de reposição hormonal, apresentando uma boa consistência interna, com um alfa de Cronbach = 0,86; na avaliação das decisões de idosos sobre a vacinação contra a gripe realizado no Canadá, com um alfa Cronbach $=0,85^{(17)}$ e na avaliação das decisões de reconstrução da mama após mastectomia, realizado nos Estados Unidos da América, sendo o questionário aplicado em inglês e espanhol ${ }^{(18)}$.

Consideramos que aumentar o entendimento da satisfação com o processo de tomada de decisão e dos fatores que a influenciam pode ajudar as enfermeiras na conceção de intervenções de apoio à decisão, razão porque julgamos importante termos instrumentos que nos possam ajudar a compreender este processo e nos motivam a desenvolver este estudo.

\section{OBJETIVO}

Descrever a adaptação cultural e a validação "The Satisfaction with Decision Scale" (1996), para o português - Portugal, analisando as propriedades psicométricas do instrumento.

\section{MÉTODO}

O processo de adaptação cultural e validação do instrumento em questão, seguiu as orientações preconizadas na literatura, orientando a sua operacionalização ${ }^{(19)}$ conforme apresentamos:

$1^{\text {a }}$ Fase - Equivalência conceitual e de itens: Iniciamos o estudo com a apreciação da equivalência conceitual, efetuando uma revisão bibliográfica sobre o tema. Foram promovidas discussões teóricas com um grupo de peritos, que considerou os itens constituintes da escala relevantes para avaliação da satisfação com as tomadas de decisão em saúde, ainda restrita em Portugal, não sendo nenhum item componente da escala original rejeitado. Foi pedida autorização da autora para tradução, adaptação e validação da escala para o português (Portugal).

$2^{\text {a }}$ Fase - Equivalência Semântica: Após ter sido obtida autorização da autora para adaptação da escala, procedemos à tradução do instrumento original. Foram realizadas duas traduções independentes, do instrumento original em inglês para o português, por profissionais com conhecimento no âmbito da saúde. As versões obtidas foram retrotraduzidas para o original, por outros tradutores, de forma independente. Foi feita avaliação formal entre as retrotraduções e o instrumento original, acompanhadas de discussão.

Efetuou-se pedido de autorização ao Presidente da Escola Superior de Enfermagem para o acesso aos estudantes da referida escola. Tendo anteriormente, sido obtido parecer Ético favorável - N. ${ }^{\circ}$ 08/CEUP/2011 da Comissão de Ética. 
Foi realizado o pré-teste, na instituição onde se iria realizar o estudo, com a aplicação da versão síntese a 20 estudantes de enfermagem, avaliando a compreensão na população alvo do estudo. Não foi necessário proceder a alterações na versão síntese. Posteriormente, os investigadores entraram em contacto com os coordenadores e/ou professores de diferentes unidades curriculares dos Cursos de Licenciatura e Mestrados, com o objetivo de solicitar a participação e colaboração no estudo.

$\mathbf{3}^{\mathbf{a}}$ Fase - Equivalência operacional: Foi respeitado o veículo da aplicação do instrumento (papel impresso) e o modo de aplicação (autopreenchimento) com o original. Os estudantes foram esclarecidos dos objetivos, finalidade de estudo e do direito de recusarem participar. Para serem elegíveis para o estudo deveriam reunir os seguintes critérios: (a) ter idade igual ou superior a 16 anos; (b) ser aluno de enfermagem (c) manifestar desejo em participar. O processo de seleção da amostra foi não probabilístico por conveniência, tendo em consideração os requisitos necessários para se proceder à análise estatística inerente à validação da escala. A colheita de dados decorreu entre 28 de Junho e 21 de Julho de 2011.

$\mathrm{O}$ instrumento de colheita de dados integrou um conjunto de questões para a obtenção dos dados socio demográficos dos estudantes e a escala "The Satisfaction with Decision Scale (SWD)"(17), composta por 6 itens que dão corpo a cada afirmação. A SWD foi concebida para avaliar a satisfação com as decisões de cuidados de saúde, sendo desenvolvida no âmbito de decisões quanto à terapia de reposição hormonal na pós-menopausa. Tal como na escala original, utilizou-se uma escala de concordância de estrutura do tipo Likert (5 opções), que varia desde discordo completamente (1) a concordo completamente (5), considerando-se que quanto maior o score obtido (score de $5=$ Alta satisfação), maior o nível de satisfação face à decisão tomada. Segundo a autora ${ }^{(17)}$, os objetivos da escala SWD são medir a satisfação global com a decisão e os três atributos de uma decisão eficaz, sendo uma vantagem da escala ser curta e fácil de usar.

$4^{\text {a }}$ Fase - Equivalência de mensuração: Nesta fase procedemos à análise das propriedades psicométricas do instrumento em estudo. Na análise estatística de dados utilizou-se o programa IBM Statistical Package for the Social Sciences (SPSS) Statistics 21 e o AMOS (v.22.0). Para além das análises descritivas, realizou-se a análise da validade de constructo, avaliação de validade dimensional e adequação de itens componentes da escala, através da análise fatorial exploratória, utilizando o método de componentes principais e avaliação de confiabilidade através da consistência interna, pelo cálculo do coeficiente alfa de Cronbach. A validade fatorial foi avaliada através da Análise Fatorial Confirmatória (AFC) com recurso ao AMOS versão 22. A existência de outliers foi avaliada pela distância quadrada de Mahalonobis e a normalidade avaliada pelo coeficiente de assimetria e curtose uni e multivariadas. Considerou-se como entrada a matriz de covariância, adotando-se o método ML (Maximum Likelihood) de estimação. A qualidade do ajustamento do modelo foi efetuada de acordo com os índices e respetivos valores de referência ${ }^{(20-21)}$. O ajustamento local foi avaliado pelos pesos fatoriais e pela fiabilidade individual dos itens. Considerou-se o Comparative Fit Index (CFI), Parcimony Comparative Fit Index (PCFI) o Tucker Lewis index (TLI), o Normed Fit Index (NFI) e, finalmente a Root Mean Square Error Approximation (RMSEA). O ajustamento do modelo teve para além dos índices de modificação as considerações teóricas. Realizamos também, o teste de validade discriminante como a autora, reunindo os itens da escala de Satisfação com a Decisão em Saúde (ESDS) com os itens da escala conceitualmente mais próxima, a Escala de Conflito da Tomada de Decisão em Saúde (ECTDS) ${ }^{(22)}$, validada para língua portuguesa ${ }^{(23)}$, que usa o mesmo modelo conceitual geral.

\section{RESULTADOS}

Para os resultados foi considerada uma probabilidade de erro máximo de $5 \%$.

Participaram no estudo 521 estudantes (42,3\%), de uma população de 1233 estudantes, maioritariamente do sexo feminino $(87,7 \% ; n=457)$, com idades compreendidas entre os 18 e os 53 anos $(M=22,5$; $D P=5,37)$. Dos participantes, $426(81,8 \%)$ frequentam o Curso de Licenciatura em Enfermagem (CLE) e 95 (18,2\%) o Mestrado. No CLE, os estudantes distribuem-se pelo $1^{\circ}$ ano $(29,1 \% ; n=124), 2^{\circ}$ ano $(23,9 \% ; n=102), 3^{\circ}$ ano $(33,6 \% ; n=143)$ e $4^{\circ}$ ano $(13,4 \%$; $\mathrm{n}=57)$, sendo que, destes, $226(53,1 \%)$; estão a frequentar a componente teórica e 200 (46,9\%) estão em estágio. Nos cursos de Mestrado, os estudantes distribuem-se pelos Mestrados em Comunitária (17,9\%), Psiquiatria (15,8\%), Médico-cirúrgica (12,6\%), Saúde Materna e Obstetrícia $(28,4 \%)$ e Reabilitação (25,3\%). São estudantes trabalhadores, no CLE, $28(6,6 \%)$, enquanto no Mestrado somente 3 (3,2\%) não são estudantes trabalhadores. Dos inquiridos, 135 $(26,0 \%)$ estão deslocados do agregado familiar, sendo, destes, 119 (28,0\%) estudantes do CLE e 16 (16,8\%) estudantes do Mestrado. Quanto à experiência de doença, 240 (56,6\%) estudantes do CLE e 55 (57,9\%) estudantes do Mestrado referiram já ter tido essa experiência. Destes, 223 (53,2\%) estudantes do CLE e 49 (52,1\%) estudantes do Mestrado tiveram que efetuar tratamento para a sua situação de doença e $156(37,2 \%)$ estudantes do CLE e 27 (29,0\%) estudantes de Mestrado tiveram de ser hospitalizados.

Com o objetivo de analisarmos a estrutura conceptual da escala, foi efetuada a Análise de Componentes Principais. $\mathrm{O}$ índice de adequação da amostra de KMO (medida de homogeneidade das variáveis) foi calculado em 0,894, sendo a matriz dos dados adequada para proceder à análise fatorial $^{(24)}$. Efetuamos uma análise fatorial exploratória de componentes principais, com rotação ortogonal dos eixos via Varimax, no sentido de maximizar a variância da matriz de pesos, para simplificar a interpretação dos fatores. Foi extraído apenas um fator que explica a variância do modelo em $63,0 \%$. No que se refere aos componentes do fator, verificamos que todas as variáveis possuem valor acima de 0,5 como se apresenta na Tabela 1. 
Tabela 1 - Comunalidade e Componentes principais da ESDS, Porto, Portugal, 2011

\begin{tabular}{lcc}
\hline \multicolumn{1}{c}{ Item } & Comunalidade & Componentes 1 \\
\hline $\begin{array}{l}\text { 6 - Estou satisfeito com a } \\
\text { minha decisão. }\end{array}$ & 0,762 & 0,873 \\
$\begin{array}{l}4 \text { - Espero realizar com } \\
\text { sucesso (ou continuar a } \\
\text { defender) a decisão que } \\
\text { tomei. }\end{array}$ & 0,712 & 0,844 \\
$\begin{array}{l}5 \text { - Estou convencido de que } \\
\text { esta era a decisão a tomar. }\end{array}$ & 0,708 & 0,841 \\
$\begin{array}{l}2 \text { - A decisão que tomei foi } \\
\text { a melhor decisão possível, } \\
\text { para mim, pessoalmente. }\end{array}$ & 0,638 & 0,799 \\
$\begin{array}{l}3 \text { - Estou convencido de } \\
\text { que a minha decisão foi } \\
\text { coerente com os meus } \\
\text { valores pessoais. }\end{array}$ & & \\
$\begin{array}{l}1 \text { - Estou satisfeito, fui } \\
\text { devidamente informado } \\
\text { sobre questões importantes } \\
\text { para a minha decisão. }\end{array}$ & 0,615 & 0,784 \\
\hline
\end{tabular}

Nota: Só um componente foi extraído. A solução não pode ser rodada.

Selecionamos os itens com carga fatorial superior a $0,3^{(24)}$. Foi calculada a consistência interna, estabelecendo-se como evidência de consistência interna satisfatória coeficientes $>0,70^{(25)}$ como se pode verificar na Tabela 2 .

Tabela 2 - Consistência Interna da ESDS, Porto, Portugal, 2011

\begin{tabular}{ll}
\hline $\begin{array}{c}\text { Itens da escala de satisfação com } \\
\text { as decisões em saúde }\end{array}$ & $\begin{array}{c}\text { Alpha } \\
\text { Cronbach }\end{array}$ \\
\hline $\begin{array}{l}\text { 6-Estou satisfeito com a minha } \\
\text { decisão }\end{array}$ &, 843 \\
$\begin{array}{l}\text { 4-Espero realizar com sucesso (ou } \\
\text { continuar a defender) a decisão que } \\
\text { tomei }\end{array}$ &, 849 \\
$\begin{array}{l}\text { 5-Estou convencido de que esta era } \\
\text { a decisão a tomar }\end{array}$ &, 849 \\
$\begin{array}{l}\text { 2-A decisão que tomei foi a melhor } \\
\text { decisão possível, para mim, } \\
\text { pessoalmente }\end{array}$ &, 857 \\
$\begin{array}{l}\text { 3-Estou convencido de que a minha } \\
\text { decisão foi coerente com os meus } \\
\text { valores pessoais }\end{array}$ \\
$\begin{array}{l}\text { 1-Estou satisfeito, fui devidamente } \\
\text { informado sobre questões } \\
\text { importantes para a minha decisão. } \\
\text { Alpha Cronbach da escala total }\end{array}$ \\
\hline
\end{tabular}

A inspeção da validade convergente foi efetuada através da correlação de cada item com a subescala ou dimensão a que pertence (escala global), através do coeficiente de correlação Pearson (r) como se pode observar na Tabela 3.

Tabela 3 - Correlação de cada item com a escala global de satisfação, Porto, Portugal, 2011

\begin{tabular}{|c|c|c|c|c|c|c|}
\hline $\begin{array}{c}\text { Satisfação } \\
\text { com a } \\
\text { decisão }\end{array}$ & Item 1 & Item 2 & Item 3 & Item 4 & Item 5 & Item 6 \\
\hline Item 2 & $445^{* *}$ & 1 & & & & \\
\hline Item 3 &, $395^{*}$ &, $570 * *$ & 1 & & & \\
\hline Item 4 &, $397 *$ &, $571 * *$ &, $615^{* *}$ & 1 & & \\
\hline Item 5 & $401 * *$ &, $592 * *$ &, $558 * *$ &, $672 * *$ & 1 & \\
\hline Item 6 &, $431 * *$ &, $628 * *$ &, $592 * *$ &, $712 * * *$ &, $735 * * *$ & 1 \\
\hline $\begin{array}{c}\text { Escala } \\
\text { Global } \\
\text { Satisfação }\end{array}$ &, $652 * *$ &, $794 * * *$ &, $783 * * *$ &, $833 * * *$ &, $836 * * *$ & $859 * * *$ \\
\hline
\end{tabular}

Nota: Correlação significativa ao nível de $p \leq 0,01$ (2-Tailled); Correlação * baixa ${ }^{* *}$ moderada $* * *$ alta

Para testar até que ponto o modelo teórico se adapta ao modelo empírico recorreu-se à análise fatorial confirmatória. Com base no modelo teórico subjacente à estrutura unidimensional testada, esta mostrou uma boa qualidade de ajustamento $\left(\mathrm{X}^{2}=2080 ; p=.000 ; \mathrm{X}^{2} / \mathrm{df}=3,334 ; \mathrm{CFI}=0,987\right.$; $\mathrm{PCFI}=0,423 ; \quad \mathrm{TLI}=0,969 \mathrm{NFI}=0,981 ; \quad \mathrm{RMSEA}=0,067$; $(\mathrm{IC} 90 \%=0,041-0,094)$. O CFI, PCFI, TLI e NFI precisam ser próximos a 0,90, enquanto o RMSEA recomendado é de até $0,08^{(20-21)}$. Os itens encontram-se moderados a fortemente saturados na variável latente com valores que variam entre 0,51 (item 1) a 0,87 (item 6) apresentando significado estatístico.

A validade discriminante foi efetuada através da analise dos componentes principais entre a escala de Satisfação com a Decisão em Saúde (ESDS) e a escala conceitualmente mais próxima (Escala de Conflito da Tomada de Decisão em Saúde - ECTDS)(23) considerando-se que a escala (ESDS) discrimina, se os itens da outra escala (ECTDS) assentam em fatores diferentes. Verificamos a existência de 4 fatores superiores a um, que, somados, explicavam a variância do modelo em $68 \%$ como se pode ver no Quadro 1.

Procedemos também à validade concorrente, efetuando a análise da correlação $r$ de Pearson, entre a escala em estudo e a escala de Conflito de Tomadas de Decisão em Saúde (ECTDS) conforme se pode observar na Tabela 4. 
Quadro 1 - Análise de componentes principais das escalas ESDS e ECTDS, variância e valores próprios ou específicos (eighenvalue) de cada fator, Porto, Portugal, 2011

\begin{tabular}{|c|c|c|c|c|}
\hline \multirow{2}{*}{$\begin{array}{l}\text { Itens das escalas - satisfação } \\
\text { com a saúde e conflito com a } \\
\text { tomada de decisão em saúde }\end{array}$} & \multicolumn{4}{|c|}{ Componentes } \\
\hline & 1 & 2 & 3 & 4 \\
\hline Item 3 Conflito decisão & ,862 & & & \\
\hline Item 2 Conflito decisão & 844 & & & \\
\hline Item 5 Conflito decisão & ,812 & & & \\
\hline Item 4 Conflito decisão & ,792 & & & \\
\hline Item 6 Conflito decisão & ,781 & & & \\
\hline Item 1 Conflito decisão & ,656 & & & \\
\hline Item 10 Conflito decisão &, 554 & & & \\
\hline Item 2 satisfação decisão & & ,778 & & \\
\hline Item 3 satisfação decisão & & ,756 & & \\
\hline Item 6 satisfação decisão & & ,745 & & \\
\hline Item 4 satisfação decisão & & ,727 & & \\
\hline Item 5 satisfação decisão & & ,684 & & \\
\hline Item 1 satisfação decisão & & 609 & & \\
\hline Item 16 Conflito decisão & & & 729 & \\
\hline Item 15 Conflito decisão & & & ,724 & \\
\hline Item 14 Conflito decisão & & & 678 & \\
\hline Item 12 Conflito decisão & & & ,616 & \\
\hline Item 13 Conflito decisão & & &, 551 & \\
\hline Item 11 Conflito decisão & & &, 523 & \\
\hline Item 8 Conflito decisão & & & & ,744 \\
\hline Item 7 Conflito decisão & & & & ,726 \\
\hline Item 9 Conflito decisão & & & & ,696 \\
\hline Eighenvalue & 10,232 & 2,590 & 1,202 & 1,034 \\
\hline Variância (total $=68,449$ ) & 46,510 & 11,773 & 5,465 & 4,701 \\
\hline
\end{tabular}

Tabela 4 - Correlação de cada item da escala ESDS com as subescalas ECTDS

\begin{tabular}{lclllll}
\hline & \multicolumn{5}{c}{ Escala de Satisfação com a Decisão em Saúde } \\
\cline { 2 - 7 } $\mathbf{r}$ & Item1 & Item2 & Item3 & Item4 & Item5 & Item6 \\
\hline ECTDS Subescala1 & $0,414^{* *}$ & $361^{*}$ & $0,359^{*}$ & $371^{*}$ & $412^{* *}$ & $0,442^{* *}$ \\
ECTDS Subescala2 & $0,423^{* *}$ & $0,503^{* *}$ & $0,497^{* *}$ & $0,626^{* *}$ & $0,627^{* *}$ & $668^{* *}$ \\
ECTDS Subescala3 & $0,438^{* *}$ & $307^{*}$ & $0,293^{*}$ & $402^{* *}$ & $0,373^{*}$ & $0,388^{* *}$ \\
\hline
\end{tabular}

*associação baixa;

** associação moderada; Correlação significativa ao nível de $p \leq 0,01$ (2-Tailled)

\section{DISCUSSÃO}

$\mathrm{Na}$ análise fatorial exploratória de componentes principais da escala ESDS, todas as variáveis dos componentes do fator, possuem valor $(\geq 0,6)$, o que indica que a variância destas variáveis é reproduzida por fatores comuns. Após a análise dos resultados obtidos (seleção dos itens com carga fatorial superior a 0,3$)$, verificou-se que estes não divergem da versão original, mantendo-se a composição da escala. A versão portuguesa, como a original, resultou num único fator, que explicam $63 \%$ da variância total, indicando que o instrumento mede a satisfação quando realizamos uma tomada de decisão em saúde. A análise correlacional entre os itens da escala e a escala global suporta esta conclusão, pois mostra que as correlações entre todos os itens e a escala global são mais fortes do que as correlações apenas entre os itens. A avaliação da fidelidade da escala variou entre 0,84 e 0,89 , verificando-se uma boa intercorrelação e homogeneidade dos itens que a compõem. Verificámos que o $\alpha$ Cronbach $=0,88$ da escala transformada apresenta valores bons e iguais à escala original (estudo piloto $\alpha$ Cronbach $=0,88$ ) e ligeiramente superiores aos estudos de terapia de reposição hormonal na pós-menopausa $\alpha$ Cronbach $=0,86$ e no estudo das decisões de pacientes idosos sobre a vacinação contra a gripe com $\alpha$ Cronbach $=0,85)^{(17)}$, o que confirma a fiabilidade da versão portuguesa.

$\mathrm{Na}$ análise fatorial confirmatória (AFC) constatou-se que a solução de 6 itens apresentada pela autora apresentava valores globais adequados de validade e fiabilidade pelo que considerámos que a escala mede adequadamente a satisfação com a decisão.

A Análise de Componentes Principais e a correlação observada entre a escala Satisfação com a Decisão em Saúde (ESDS) e escala de Conflito de Tomadas de Decisão em Saúde (ECTDS) indica que as escalas tem construções que podem ser consideradas distintas embora ambas as escalas estejam relacionadas.

A nomeação adotada na versão portuguesa procurou ir de encontro à denominação da escala original. Consideramos como pontos fortes deste estudo o número de participantes $(N=521)$, tendo também sido ultrapassado o número necessário ao recomendado na literatura para se proceder à análise fatorial ${ }^{(24)}$. No que se refere às características demográficas, tal como na realidade dos estudantes do Ensino Superior em Portugal, observou-se um grande predomínio do género feminino face ao masculino, sendo a média das idades (22,5 anos) próxima à média nacional (23 anos), o que torna mais forte a possibilidade de generalizar os resultados. Relativamente à tomada de decisão nas opções terapêuticas da síndrome gripal, verificamos não existirem diferenças significativas entre os alunos do CLE e Mestrado, já que ambos optam pelo controle sintomatológico como primeira opção (75,8\% dos alunos do CLE e 59,4\% do Mestrado), seguidos das opções "Medidas de etiqueta respiratória e distanciamento social" (14,3\% dos alunos do CLE e 19,8\% do Mestrado) e da "Vacinação" (6,6\% dos alunos do CLE e 16,7\% do Mestrado). 
Ambos os alunos se sentem inseguros quanto às opções $(3,3 \%$ dos alunos do CLE e 3,1\% do Mestrado). No entanto, as opções que reúnem maior nível de concordância, relativamente à satisfação com a tomada de decisão, são as "Medidas de etiqueta respiratória e distanciamento social", para 91,8\% dos estudantes do CLE, e a "Vacinação", para 100\% dos estudantes do Mestrado. $\mathrm{O}$ item que reúne maior nível de concordância na opção "Medidas de etiqueta respiratória e distanciamento social" é o item 1 (satisfação com a informação), sugerindo que estes alunos têm mais informação acerca das opções de que dispõem. Na opção "Vacinação", os itens 2,3,4 e 6 (satisfação com a perspetiva e os valores pessoais e com a manutenção da decisão no futuro), são os que reúnem maior nível de concordância, o que nos sugere que aumentar o envolvimento das pessoas na decisão, melhorar a informação/conhecimento e a perceção realista dos resultados ${ }^{(26-28)}$, é basilar para se fazer escolhas congruentes com as necessidades e expectativas de cada pessoa. Estas ações são tradutoras de um maior nível de satisfação com a decisão tomada, resultado congruente com alguns dos vários estudo publicados ${ }^{(5-8)}$. Não se verificaram diferenças significativas no estudo das relações entre as restantes variáveis demográficas com as dimensões da escala portuguesa. Apesar da opção "controle sintomatológico recorrendo a fármacos" ser a mais escolhida, como atitude terapêutica perante a síndrome gripal esta opção é a que reúne menor nível de concordância nos itens 5, 1 e 3. Sugerindo-nos menor convicção na escoIha realizada, menor conhecimento/informação obtido para suportar a decisão a tomar, menor sentido de congruência da escolha com os valores pessoais e menor previsibilidade de manutenção da escolha. Estes dados vêm reforçar a importância de realizar estudos que nos permitam compreender a satisfação e o processo de decisão em saúde. Julgamos que este estudo é inovador porque o processo de decisão sob a ótica do paciente ainda é pouco estudado e dispor de um instrumento que possibilite ampliar esta compreensão aliado à perceção de satisfação é relevante para o conhecimento em saúde.

\section{CONCLUSÕES}

A "Escala de Satisfação com a Decisão em Saúde - ESDS", que resultou da adaptação transcultural da escala Americana "The Satisfaction with Decision Scale (SWD)", reúne critérios de validade psicométrica, sendo um instrumento confiável, válido e promissor para a avaliação da satisfação na tomada de decisão em saúde.

Consideramos também, que pode ser bastante útil como complemento na investigação, contribuindo para avaliar a satisfação global com as decisões em saúde e para conhecer melhor os fatores intervenientes na tomada de decisão em saúde.

\section{AGRADECIMENTO}

Agradecemos à Profa Doutora Teresa Martins o auxilio dado na análise estatística confirmatória.

\section{REFERÊNCIAS}

1. Mishima SM, Pereira FH, Matumoto S, Fortuna CM, Fortuna $C M$, Pereira MJB, et al. A assistência na saúde da família sob a perspectiva dos usuários. Rev Latinoam Enferm. 2010;18(3):148-55.

2. Paiva SMA, Gomes ELR. Assistência hospitalar: avaliação da satisfação dos usuários durante seu período de internação. Rev Latinoam Enferm. 2007;15(5):973-9.

3. Silva Júnior JF, Araújo SP, Nava AV, Dias RS. Serviço prestado em uma clínica escola: fatores influentes na satisfação dos pacientes. Rev Saúde Pesquisa. 2014;7(1):129-34.

4. Cacique DB, Bacha AM, Grassioto OR, Oliveira HC, Lima MT, Fonsechi-Carvasan GA. A avaliação da satisfação de pacientes internadas em um hospital universitário especializado na saúde materno-infantil: questionários e resultados globais. Rev Adm Saúde [Internet]. 2012 [acesso em 23 de novembro de 2008];14(57):134-42. Disponível em: file:///C:/ Users/Cliente/Downloads/RAS_v14n57_134-142.pdf

5. Johannessen G, Eikeland A, Stubberud DG, Fagerstöm L. A descriptive study of patient satisfaction and the structural factors of Norwegian intensive care nursing. Intensive Crit Care Nurs. 2011;27(5):281-9.

6. OConnor AM, Wennberg JE, Legare F, Llewellyn-Thomas HA, Moulton BW, Sepucha KR, et al. Toward the 'tipping point': decision aids and informed patient choice. Health Aff. 2007;26(3):716-25.
7. Duffy JR, Hoskins LM. The quality-caring model: blending dual paradigms. ANS Adv Nurs Sci. 2003;26(1):77-88.

8. Budden LM, Hayes BA, Pierce PF, Buether PG. Australian women`s prediagnostic values and influencing sociodemographic variables relating to treatment choices for early breast cancer treatment. Australian Journal Cancer Nursing [Internet]. 2007 [cited 2008 November 23];8(1):9-20. Available from: http://researchonline.jcu.edu.au/2138/2/ Budden_et_al_2007.pdf

9. Wagner D, Bear M. Patient satisfaction with nursing care: a concept analysis within a nursing framework. J Adv Nurs [Internet]. 2009 [cited 2008 November 23];65(3):692701. Available from: http://www.ncbi.nlm.nih.gov/ pubmed/19016924

10. Schmidt SMS, Müller FM, Santos E, Ceretta PS, Garlet V, Schmitt S. Análise da satisfação dos usuários em um hospital universitário. Saúde Debate [Internet]. 2014 [acesso em 23 de novembro de 2008];38(101):305-17. Disponível em: http://www.scielo.br/pdf/sdeb/v38n101/01031104-sdeb-38-101-0305.pdf

11. Sorlei V, Torjuul K, Ross A, Kihlgren M. Satisfied patients are also vulnerable patients-narratives from an acute care ward. J Clin Nurs. 2006;15(10):1240-6.

12. Pierce PF. Deciding on breast cancer treatment: adescription of decision behavior. Nurs Res. 1993;42(1):22-8. 
13. Huey-Ming T, Chang-Yi Y. Patient satisfaction versus quality. Nurs Ethics. 2008;15(1):121-4.

14. Tervo-Heikkinen T, Kvist R, Partanen P, VehvilãinenJulkunen K, Aato P. Patient satisfaction as a positive nursing outcome. J Nurs Care Qual. 2008;23(1):58-65.

15. Sahin B, Yilmaz F, Lee K. Factor's affecting inpatient satisfaction: structural equation modeling. J Med. Syst. 2007;31(1):9-16.

16. Gil L, White L. A critical review of patient satisfaction. Leadersh Health Serv. 2009;22(1):8-19.

17. Holmes-Rovner M, Kroll J, Schmitt N, Rovner DR, Breer $M L$, Rothert ML, et al. Patient satisfaction with health care decisions: the satisfaction with decision scale. Med Decis Making. 1996;16(1):56-64.

18. Alderman AK, Hawley ST, Morrow M, Salem B, Hamilton A, Graff JJ, et al. Receipt of delayed breast reconstruction after mastectomy: do women revisit the decision? Ann Surg Oncol. 2011;18(6):1748-56.

19. Reichenheim ME, Moraes CL. Operacionalização de adaptação transcultural de instrumentos de aferição usados em epidemiologia. Rev Saúde Pública [Internet]. 2007 [acesso em 23 de novembro de 2008];41(4):1-8. Disponível em: www.scielo.br/pdf/rsp/nahead/6294.pdf

20. Kline RB. Principles and practice of structural equation modeling. $3^{\text {th }}$ ed. New York: The Guilford presss; 2011.

21. Maroco J. Análise de Equações Estruturais: fundamentos teóricos, software \& aplicações. Pêro Pinheiro (PT): Report Number; 2010.

22. O`Connor AM. Validation of a decision conflicts scale. Med Decis Making. 1995;15(1):25-30.

23. Martinho MJCM, Martins MFPSM, Angelo M. Escala de conflito em tomadas de decisão em saúde: instrumento adaptado e validado para língua portuguesa. Rev Esc Enferm USP. 2013;47(3):576-83.

24. Figueiredo Filho DB, Silva Júnior JA. Visão além do alcance: uma introdução à análise fatorial. Opin Pública. 2010;16(1):160-85.

25. DeVon HA, Block ME, Moyle-Wright P, Ernst DM, Hayden SJ, Lazzara DJ, et al. A psychometric toolbox for testing validity and reliability. J Nurs Scholarsch. 2007;39(2):155-64.

26. Elwyn G,Frosch D, Volandes AE, Edwards A, Montori VM. Investing in deliberation: a definition and classification of decision support interventions for people facing difficult health decisions. Med Decis Making. 2010;30(6):701-11.

27. Stacey D, Légaré $F$, Col NF, Bennett CL, Barry MJ, Eden $\mathrm{KB}$, et al.Decision aids for people facing health treatment or screening decisions. Cochrane Database Syst Rev.2011;(10):CD001431.

28. Gentles SJ, Stacey D, Bennett C, Alshurafa M, Walter SD. Factors explaining the heterogeneity of effects of patient decision aids on knowledge of outcome probabilities: a systematic review sub-analysis. Syst Rev. 2013;2:95. 